\title{
Captures
}

Figures, théories et pratiques de l'imaginaire

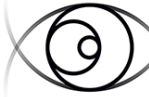

C A P T U R E S

\section{Volume 2 Numéro 1}

\section{Vincent Lavoie}

Volume 2, Number 1, 2017

\section{Écrivains à l’écran}

URI: https://id.erudit.org/iderudit/1059796ar

DOI: https://doi.org/10.7202/1059796ar

See table of contents

Publisher(s)

Figura, Centre de recherche sur le texte et l'imaginaire

ISSN

2371-1930 (digital)

Explore this journal

Cite this document

Lavoie, V. (2017). Volume 2 Numéro 1. Captures, 2(1).

https://doi.org/10.7202/1059796ar

\section{Article abstract}

Quelle visualité pour la figure de l'écrivain? À cette question, le cinéma et les séries télévisées ont fourni un ensemble très diversifié de réponses, allant de la représentation littérale de l'acte d'écriture à la mise en œuvre des fictions littéraires de l'auteur, en passant par la monstration de ses déboires sentimentaux ou de sa construction médiatique. Le dossier « Écrivains à l'écran » prend en compte un éventail de films entrelaçant éthos du réalisateur et de...
This document is protected by copyright law. Use of the services of Erudit (including reproduction) is subject to its terms and conditions, which can be viewed online.

https://apropos.erudit.org/en/users/policy-on-use/ 
Version enrichie de cet article : http://revuecaptures.org/node/832

\section{Volume 2 Numéro 1}

\section{Vincent Lavoie}

Résumé :

Quelle visualité pour la figure de l'écrivain? À cette question, le cinéma et les séries télévisées ont fourni un ensemble très diversifié de réponses, allant de la représentation littérale de l'acte d'écriture à la mise en œuvre des fictions littéraires de l'auteur, en passant par la monstration de ses déboires sentimentaux ou de sa construction médiatique. Le dossier “ Écrivains à l'écran » prend en compte un éventail de films entrelaçant éthos du réalisateur et de...

Quelle visualité pour la figure de l'écrivain? À cette question, le cinéma et les séries télévisées ont fourni un ensemble très diversifié de réponses, allant de la représentation littérale de l'acte d'écriture à la mise en œuvre des fictions littéraires de l'auteur, en passant par la monstration de ses déboires sentimentaux ou de sa construction médiatique. Les «Écrivains à l'écran », thème du dossier dirigé par Nadja Cohen, prend en compte un éventail de films entrelaçant éthos du réalisateur et de l'écrivain, narration visuelle et écriture, écran et page. Preuve de l'ampleur du phénomène, celui-ci concerne tous les genres : mélodrames, films policiers, thrillers, giallos, films d'horreur, films érotiques ou films d'aventures. Les huit contributions réunies dans le présent dossier décryptent ces riches intrications au prisme des études culturelles, littéraires et cinématographiques. Qu'ils soient réels ou fictifs, les écrivains en question - Houellebecq ou Baudelaire, Hank Moody ou George Stark — s'imposent à l'écran sous la forme de « projections » imaginaires, même lorsque celles-ci flirtent avec le genre documentaire du biopic. Telle est l'une des lignes de force des "Écrivains à l'écran », prolongée par les propositions artistiques et littéraires rassemblées dans la section «Contrepoints ». Fictions identitaires et mythifications des « monstres sacrés » de la littérature (Emilio LópezMenchero), palimpsestes de gestes artistiques (Virgile Loyer), pugilat amusé entre écriture et production cinématographique (Vincent Ravalec), écrivain vlogeur (François Bon) ou encore aporie de l'enregistrement de l'écriture (Marcel Broodthaers) figurent au nombre de ces prolongements.

Dans la rubrique « Hors dossier », la spécialiste en philosophie de l'environnement, Virginie Maris, en appelle à une critique fondamentale du caractère colonialiste, patriarcal et anthropocentriste de la société contemporaine à partir de l'analyse de la récente exposition produite par le Centre canadien d'architecture (CCA), Le temps presse. Une contre-histoire environnementale du Canada moderne, une interrogation engagée des rapports entretenus par le Canada avec sa nature.

Enfin, la signature visuelle de ce numéro a été confiée à l'artiste québécoisMartin Beauregard, dont la série 
photographique Drive End(2007-2011) met en exergue un imaginaire de la fin du cinéma.

Vincent Lavoie

Directeur 